\title{
Existence Results for Systems of Quasi-Variational Relations
}

\author{
DANIELA IOANA INOAN*
}

ABSTRACT. The existence of solutions for a system of variational relations, in a general form, is studied using a fixed point result for contractions in metric spaces. As a particular case, sufficient conditions for the existence of solutions of a system of quasi-equilibrium problems are given.

Keywords: Variational relations problems, system of equilibrium problems, fixed points.

2010 Mathematics Subject Classification: 47H09, 58E99.

\section{INTRODUCTION AND PRELIMINARIES}

For each $i \in I=\{1, \ldots, n\}$, let $X_{i}$ be a nonempty subset of a complete metric space $\left(E_{i}, d_{i}\right)$ and $X=\prod_{i \in I} X_{i}$ a subset of the product space $E=\prod_{i \in I} E_{i}$. Let $S_{i}, Q_{i}: X \rightarrow 2^{X_{i}}$ be two set-valued maps with nonempty values. Let $R_{i}\left(x, y_{i}\right)$ be a relation between $x \in X$ and $y_{i} \in X_{i}$.

The general system of quasi-variational relations that we consider in this paper is:

$(S Q V R)$ Find $\bar{x}=\left(\bar{x}_{1}, \ldots, \bar{x}_{n}\right) \in X$ such that for each $i \in I$,

$$
\bar{x}_{i} \in S_{i}(\bar{x}) \text { and } R_{i}\left(\bar{x}, y_{i}\right) \text { holds for all } y_{i} \in Q_{i}(\bar{x}) \text {. }
$$

Variational relations problems were considered for the first time by D.T. Luc in [11], as a general model that encompasses optimization problems, equilibrium problems or variational inclusion problems. Several authors continued the study of variational relations problems, see for instance the papers [10], [12], [9], [2], [1] and the references therein. Existence results for the solutions of variational relations problems are obtained mostly in two ways: by applying intersection results for set valued mappings (see [11]) or by using various fixed points theorems (see [11], [7], [4]).

The system $(S Q V R)$ was introduced by L.J. Lin and Q.H. Ansari in [8], where the existence of a solution was established using a maximal element theorem for a family of set-valued maps. The same system was studied in [5] by a factorization method, that followed the ideas from [6].

In this paper, we will give sufficient conditions for the existence of solutions of the system $(S Q V R)$, using a fixed-point theorem for set-valued mappings that are Reich-type contractions. The general result obtained for the system of variational relations will be applied in the last section to a system of equilibrium problems.

In the rest of this section, we present some notations and results needed in the paper.

The metric on the product space will be defined by $d: E \times E \rightarrow \mathbb{R}_{+}$,

$$
d(x, y)=d_{1}\left(x_{1}, y_{1}\right)+\cdots+d_{n}\left(x_{n}, y_{n}\right)
$$

Received: 5 October 2019; Accepted: 20 November 2019; Published Online: 23 November 2019

*Corresponding author: Daniela Ioana Inoan; daniela.inoan@math.utcluj.ro

DOI: $10.33205 / \mathrm{cma} .643397$ 
for $x=\left(x_{1}, \ldots, x_{n}\right) \in E$ and $y=\left(y_{1}, \ldots, y_{n}\right) \in E$.

For any nonempty sets $A, B \subset E$ and $x \in E$, denote by

$$
\begin{aligned}
D(x, B) & =\inf _{b \in B} d(x, b) \text { and } \\
H(A, B) & =\max \left\{\sup _{a \in A} \inf _{b \in B} d(a, b), \sup _{b \in B} \inf _{a \in A} d(a, b)\right\} .
\end{aligned}
$$

$H(A, B)$ is the generalized Hausdorff functional of $A$ and $B$. Similarly, we will denote by $H_{i}\left(A_{i}, B_{i}\right)$ the Hausdorff distance induced by $d_{i}$, for $A_{i}$ and $B_{i}$ subsets of $E_{i}$.

Lemma 1.1. For $x=\left(x_{1}, \ldots, x_{n}\right), A=A_{1} \times \cdots \times A_{n}$ and $B=B_{1} \times \cdots \times B_{n}$, we have

$$
\begin{aligned}
D(x, B) & =D_{1}\left(x_{1}, B_{1}\right)+\cdots+D_{n}\left(x_{n}, B_{n}\right), \\
H(A, B) & \leq H_{1}\left(A_{1}, B_{1}\right)+\cdots+H_{n}\left(A_{n}, B_{n}\right) .
\end{aligned}
$$

Lemma 1.2. (a) If $A, B \subset E$ are such that for each $a \in A$ there exists $b \in B$ such that $d(a, b) \leq c$ and for each $b \in B$ there exists $a \in A$ such that $d(a, b) \leq c$, then $H(A, B) \leq c$.

(b) If $A, B \subset E$ and $\varepsilon>0$, then for each $a \in A$ there exists $b \in B$ such that $d(a, b) \leq H(A, B)+\varepsilon$.

There is a vast literature on the existence of fixed points of generalized contractions, both single-valued and set-valued (see for instance [3], [14]). We will use the following:

A set-valued mapping $F: E \rightarrow 2^{E}$ is said to be a Reich - type contraction if there exist $a, b, c \geq 0$, with $a+b+c<1$ such that $H(F(x), F(y)) \leq a d(x, y)+b D(x, F(x))+c D(y, F(y))$, for each $x, y \in E$.

Theorem 1.1 ([13]). Let $(E, d)$ be a complete metric space and let $F: E \rightarrow 2^{E}$ be a Reich-type contraction. Suppose also that $F(x)$ is a closed set, for every $x \in E$. Then, $F$ has at least a fixed point.

\section{AN EXISTENCE RESULT FOR A SYSTEM OF VARIATIONAL RELATIONS}

We give in what follows sufficient conditions for the existence of solutions of the system $(S Q V R)$ formulated in the previous section.

For $x=\left(x_{1}, \ldots, x_{n}\right) \in X$ and $i \in I$ fixed, we denote

$$
\Gamma_{i}(x)=\left\{z_{i} \in S_{i}(x) \mid R_{i}\left(x_{1}, \ldots, z_{i}, \ldots, x_{n} ; t_{i}\right) \text { holds for all } t_{i} \in Q_{i}(x)\right\}
$$

and we define the function $\Gamma: X \rightarrow 2^{X}$ by $\Gamma(x)=\Gamma_{1}(x) \times \cdots \times \Gamma_{n}(x)$. It is easy to see that any fixed point of $\Gamma$ is a solution of (SQVR).

Theorem 2.2. Suppose that for any $i \in I$, the set $X_{i}$ is nonempty, closed and:

(i) for any $x \in X, \Gamma_{i}(x)$ is nonempty;

(ii) there exists $\left.q_{i} \in\right] 0,1\left[\right.$ such that, for every $x^{1}, x^{2} \in X$, if $z_{i}^{1} \in \Gamma_{i}\left(x^{1}\right)$, there exists $z_{i}^{2} \in \Gamma_{i}\left(x^{2}\right)$ such that

$$
d_{i}\left(z_{i}^{1}, z_{i}^{2}\right) \leq q_{i} H_{i}\left(S_{i}\left(x^{1}\right), S_{i}\left(x^{2}\right)\right) ;
$$

(iii) there exist $\left.a_{i}, b_{i}, c_{i} \in\right] 0,1\left[\right.$, with $\max _{i \in I} a_{i}+\max _{i \in I} b_{i}+\max _{i \in I} c_{i}<1$ such that, for every $x^{1}, x^{2} \in X$,

$$
H_{i}\left(S_{i}\left(x^{1}\right), S_{i}\left(x^{2}\right)\right) \leq a_{i} d_{i}\left(x_{i}^{1}, x_{i}^{2}\right)+b_{i} D_{i}\left(x_{i}^{1}, S_{i}\left(x^{1}\right)\right)+c_{i} D_{i}\left(x_{i}^{2}, S_{i}\left(x^{2}\right)\right) ;
$$

(iv) for any $x \in X$, the set $S_{i}(x)$ is closed;

(v) the relation $R_{i}$ is closed in the $i-t h$ variable, that is: for any sequence $\left(z_{i}^{k}\right)_{k \in \mathbb{N}} \subset X_{i}$ such that $z_{i}^{k} \rightarrow z_{i}$ when $k \rightarrow \infty$, if $R_{i}\left(x_{i}, \ldots, z_{i}^{k}, \ldots x_{n} ; t_{i}\right)$ holds, then $R_{i}\left(x_{i}, \ldots, z_{i}, \ldots x_{n} ; t_{i}\right)$ holds too.

Then, (SQVR) admits at least a solution. 
Proof. We will prove that $\Gamma: X \rightarrow 2^{X}$ is a Reich-type contraction and we will use Theorem 1.1 to obtain the existence of a fixed point of $\Gamma$. Since $X$ is closed and $(E, d)$ is complete, the space $(X, d)$ is complete too.

For each $i \in I$ and $x \in X$, hypotheses (iv) and (v) imply that $\Gamma_{i}(x)$ is closed. Then $\Gamma(x)$ is closed too.

Let $x^{1}=\left(x_{1}^{1}, \ldots, x_{n}^{1}\right) \in X$ and $x^{2}=\left(x_{1}^{2}, \ldots, x_{n}^{2}\right) \in X$. Let $z_{i}^{1} \in \Gamma_{i}\left(x^{1}\right)$. According to (ii), there exists $z_{i}^{2} \in \Gamma_{i}\left(x^{2}\right)$ such that

$$
d_{i}\left(z_{i}^{1}, z_{i}^{2}\right) \leq q_{i} H_{i}\left(S_{i}\left(x^{1}\right), S_{i}\left(x^{2}\right)\right) .
$$

Similarly, for any $z_{i}^{2} \in \Gamma_{i}\left(x^{2}\right)$ there exists $z_{i}^{1} \in \Gamma_{i}\left(x^{1}\right)$ such that (2.1) holds. From Lemma 1.2, we have

$$
H_{i}\left(\Gamma_{i}\left(x^{1}\right), \Gamma_{i}\left(x^{2}\right)\right) \leq q_{i} H_{i}\left(S_{i}\left(x^{1}\right), S_{i}\left(x^{2}\right)\right) .
$$

Further, using Lemma 1.1, (2.2), (iii), and the inclusion $\Gamma_{i}(x) \subseteq S_{i}(x)$, for any $x \in X$, follows

$$
\begin{aligned}
H\left(\Gamma\left(x^{1}\right), \Gamma\left(x^{2}\right)\right) & \leq \sum_{i=1}^{n} H_{i}\left(\Gamma_{i}\left(x^{1}\right), \Gamma_{i}\left(x^{2}\right)\right) \leq \sum_{i=1}^{n} q_{i} H_{i}\left(S_{i}\left(x^{1}\right), S_{i}\left(x^{2}\right)\right) \\
& \leq \sum_{i=1}^{n}\left(q_{i} a_{i} d_{i}\left(x_{i}^{1}, x_{i}^{2}\right)+q_{i} b_{i} D_{i}\left(x_{i}^{1}, S_{i}\left(x^{1}\right)\right)+q_{i} c_{i} D_{i}\left(x_{i}^{2}, S_{i}\left(x^{2}\right)\right)\right) \\
& \leq q a d\left(x^{1}, x^{2}\right)+q b \sum_{i=1}^{n} D_{i}\left(x_{i}^{1}, \Gamma_{i}\left(x^{1}\right)\right)+q c \sum_{i=1}^{n} D_{i}\left(x_{i}^{2}, \Gamma_{i}\left(x^{2}\right)\right) \\
& =q a d\left(x^{1}, x^{2}\right)+q b D\left(x^{1}, \Gamma\left(x^{1}\right)\right)+q c D\left(x^{2}, \Gamma\left(x^{2}\right)\right),
\end{aligned}
$$

where $q=\max _{i \in I} q_{i}, a=\max _{i \in I} a_{i}, b=\max _{i \in I} b_{i}, c=\max _{i \in I} c_{i}$ and $q a+q b+q c<1$. Applying Reich's theorem follows the existence of a fixed point for $\Gamma$ and consequently of a solution of (SQVR).

By making a change in hypothesis (ii), we can obtain a second existence result:

Theorem 2.3. Suppose that for any $i \in I$, the set $X_{i}$ is nonempty, closed and:

(i) for any $x \in X, \Gamma_{i}(x)$ is nonempty;

(ii) there exists $\left.q_{i} \in\right] 0,1\left[\right.$ such that, for every $x^{1}, x^{2} \in X$, for every $z_{i}^{1} \in \Gamma_{i}\left(x^{1}\right)$ and $z_{i}^{2} \in \Gamma_{i}\left(x^{2}\right)$,

$$
d_{i}\left(z_{i}^{1}, z_{i}^{2}\right) \leq q_{i} H_{i}\left(S_{i}\left(x^{1}\right), S_{i}\left(x^{2}\right)\right) ;
$$

(iii) there exist $\left.a_{i}, b_{i}, c_{i} \in\right] 0,1\left[\right.$, with $\max _{i \in I} a_{i}+\max _{i \in I} b_{i}+\max _{i \in I} c_{i}<1$ such that, for every $x^{1}, x^{2} \in X$,

$$
H_{i}\left(S_{i}\left(x^{1}\right), S_{i}\left(x^{2}\right)\right) \leq a_{i} d_{i}\left(x_{i}^{1}, x_{i}^{2}\right)+b_{i} D_{i}\left(x_{i}^{1}, S_{i}\left(x^{1}\right)\right)+c_{i} D_{i}\left(x_{i}^{2}, S_{i}\left(x^{2}\right)\right) ;
$$

Then, (SQVR) admits a solution.

Proof. It can be noticed that for any $x \in X$ and $i \in I$, the set $\Gamma_{i}(x)$ contains only one element. Indeed, if $\zeta_{i}, \xi_{i} \in \Gamma_{i}(x)$, according to (ii), we get

$$
d_{i}\left(\zeta_{i}, \xi_{i}\right) \leq q_{i} H_{i}\left(S_{i}(x), S_{i}(x)\right)=0,
$$

so $\zeta_{i}=\xi_{i}$. Since $\Gamma_{i}(x)$ is a singleton, it is a closed set. The rest of the proof is the same as for Theorem 2.2.

Starting with another definition for the "partial" problem, we can obtain a new existence result, with different conditions. 
For $x=\left(x_{1}, \ldots, x_{n}\right) \in X$ and $i \in I$ fixed, we denote

$$
\begin{aligned}
T_{i}(x)=\left\{z_{i} \in X_{i} \mid \quad z_{i}\right. & \in S_{i}\left(x_{1}, \ldots, z_{i}, \ldots, x_{n}\right) \text { and } R_{i}\left(x_{1}, \ldots, z_{i}, \ldots, x_{n} ; \theta_{i}\right) \\
& \text { holds for all } \left.\theta_{i} \in Q_{i}\left(x_{1}, \ldots, z_{i}, \ldots, x_{n}\right)\right\}
\end{aligned}
$$

and we define the function $T: X \rightarrow 2^{X}$ by $T(x)=T_{1}(x) \times \cdots \times T_{n}(x)$. It is easy to see that any fixed point of $T$ is a solution of (SQVR).

Theorem 2.4. Suppose that for any $i \in I$, the set $X_{i}$ is nonempty, closed and:

(i) for any $x \in X, T_{i}(x)$ is nonempty;

(ii) there exists $\left.q_{i} \in\right] 0,1\left[\right.$ such that, for every $x^{1}, x^{2} \in X$, if $z_{i}^{1} \in T_{i}\left(x^{1}\right)$, there exists $z_{i}^{2} \in T_{i}\left(x^{2}\right)$ such that

$$
d_{i}\left(z_{i}^{1}, z_{i}^{2}\right) \leq q_{i} H_{i}\left(S_{i}\left(x^{1}\right), S_{i}\left(x^{2}\right)\right)
$$

(iii) there exist $\left.a_{i}, b_{i}, c_{i} \in\right] 0,1\left[\right.$, with $\max _{i \in I} a_{i}+\max _{i \in I} b_{i}+\max _{i \in I} c_{i}<1$ such that, for every $x^{1}, x^{2} \in X$,

$$
H_{i}\left(S_{i}\left(x^{1}\right), S_{i}\left(x^{2}\right)\right) \leq a_{i} d_{i}\left(x_{i}^{1}, x_{i}^{2}\right)+b_{i} D_{i}\left(x_{i}^{1}, S_{i}\left(x^{1}\right)\right)+c_{i} D_{i}\left(x_{i}^{2}, S_{i}\left(x^{2}\right)\right) ;
$$

(iv) for any sequence $\left(z_{i}^{k}\right)_{k \in \mathbb{N}} \subset X_{i}$ such that $z_{i}^{k} \rightarrow z_{i}$ when $k \rightarrow \infty$, if $z_{i}^{k} \in S_{i}\left(x_{1}, \ldots, z_{i}^{k}, \ldots, x_{n}\right)$ for any $k \in \mathbb{N}$, then $z_{i} \in S_{i}\left(x_{1}, \ldots, z_{i}, \ldots, x_{n}\right)$;

(v) for any sequence $\left(z_{i}^{k}\right)_{k \in \mathbb{N}} \subset X_{i}$ such that $z_{i}^{k} \rightarrow z_{i}$ when $k \rightarrow \infty$, if $R_{i}\left(x_{i}, \ldots, z_{i}^{k}, \ldots x_{n} ; \theta_{i}\right)$ holds for any $\theta_{i} \in Q_{i}\left(x_{1}, \ldots, z_{i}^{k}, \ldots, x_{n}\right)$, then the relation $R_{i}\left(x_{i}, \ldots, z_{i}, \ldots x_{n} ; t_{i}\right)$ holds for any $t_{i} \in Q_{i}\left(x_{1}, \ldots, z_{i}, \ldots, x_{n}\right)$.

Then, (SQVR) admits at least a solution.

Proof. Hypotheses (iv) and (v) imply that for every $x \in X, T(x)$ is closed. The rest of the proof is identical to the one of Theorem 2.2.

\section{AN EXISTENCE RESULT FOR A SYSTEM OF QUASI-EQUILIBRIUM PROBLEMS}

As a particular case of the system of quasi-variational relations, we consider

$(S Q E P)$ Find $\bar{x}=\left(\bar{x}_{1}, \ldots, \bar{x}_{n}\right) \in X$ such that for each $i \in I$,

$$
\bar{x}_{i} \in S_{i}(\bar{x}) \text { and } f_{i}\left(\bar{x}, t_{i}\right) \geq 0 \text { for all } t_{i} \in S_{i}(\bar{x}) .
$$

The relation $R_{i}\left(x, t_{i}\right)$ holds iff $f_{i}\left(x, t_{i}\right) \geq 0$. In this section, we denote

$$
\gamma_{i}(x)=\left\{z_{i} \in S_{i}(x) \mid f_{i}\left(x_{1}, \ldots, z_{i}, \ldots, x_{n} ; t_{i}\right) \geq 0, \text { for all } t_{i} \in S_{i}(x)\right\}
$$

As a consequence of Theorem 2.3, we obtain:

Theorem 3.5. Suppose that for any $i \in I$, the set $X_{i}$ is nonempty, closed and:

(a) for any $x \in X, \gamma_{i}(x)$ is nonempty;

(b) there exist $\left.a_{i}, b_{i}, c_{i} \in\right] 0,1\left[\right.$, with $\max _{i \in I} a_{i}+\max _{i \in I} b_{i}+\max _{i \in I} c_{i}<1$ such that, for every $x^{1}, x^{2} \in X$,

$$
H_{i}\left(S_{i}\left(x^{1}\right), S_{i}\left(x^{2}\right)\right) \leq a_{i} d_{i}\left(x_{i}^{1}, x_{i}^{2}\right)+b_{i} D_{i}\left(x_{i}^{1}, S_{i}\left(x^{1}\right)\right)+c_{i} D_{i}\left(x_{i}^{2}, S_{i}\left(x^{2}\right)\right) ;
$$

(c) there exists $m_{i}>0$ such that for every $x=\left(x_{1}, \ldots, x_{n}\right) \in X$ and $t_{i} \in X_{i}$,

$$
f_{i}\left(x_{1}, \ldots, x_{i}, \ldots, x_{n} ; t_{i}\right)+f_{i}\left(x_{1}, \ldots, t_{i}, \ldots, x_{n} ; x_{i}\right) \leq-m_{i} d_{i}\left(x_{i}, t_{i}\right) ;
$$

(d) $f_{i}$ is lipschitz in the last variable, that is there exists $L_{i}>0$ such that for every $x \in X$ and $t_{i}, \theta_{i} \in X_{i}$,

$$
\left|f_{i}\left(x ; t_{i}\right)-f_{i}\left(x ; \theta_{i}\right)\right| \leq L_{i} d_{i}\left(t_{i}, \theta_{i}\right)
$$


(e) $f_{i}$ is lipschitz in the $i-t h$ variable, that is there exists $\lambda_{i}>0$ such that for every $x \in X$ and $\zeta_{i}, \xi_{i}, t_{i} \in X_{i}$

$$
\left|f_{i}\left(x_{1}, \ldots, \zeta_{i}, \ldots x_{n} ; t_{i}\right)-f_{i}\left(x_{1}, \ldots, \xi_{i}, \ldots x_{n} ; t_{i}\right)\right| \leq \lambda_{i} d_{i}\left(\zeta_{i}, \xi_{i}\right)
$$

(f) $L_{i}+\lambda_{i}<m_{i}$.

Then, (SQEP) admits a solution.

Proof. To apply Theorem 2.3 , we just need to verify hypothesis (ii). Let $\varepsilon>0$. Let $x^{1}, x^{2} \in X$ and $z_{i}^{1} \in \gamma\left(x^{1}\right), z_{i}^{2} \in \gamma\left(x^{2}\right)$.

Since $z_{i}^{1} \in S_{i}\left(x^{1}\right)$, from Lemma 1.2, there exists $t_{i}^{2} \in S_{i}\left(x^{2}\right)$ such that

$$
d_{i}\left(z_{i}^{1}, t_{i}^{2}\right) \leq H_{i}\left(S_{i}\left(x^{1}\right), S_{i}\left(x^{2}\right)\right)+\varepsilon .
$$

Similarly, since $z_{i}^{2} \in S_{i}\left(x^{2}\right)$, there exists $t_{i}^{1} \in S_{i}\left(x^{1}\right)$ such that

$$
d_{i}\left(z_{i}^{2}, t_{i}^{1}\right) \leq H_{i}\left(S_{i}\left(x^{1}\right), S_{i}\left(x^{2}\right)\right)+\varepsilon .
$$

From the definitions of $\gamma_{i}\left(x^{1}\right)$ and $\gamma_{i}\left(x^{2}\right)$, we get

$$
f_{i}\left(x_{1}^{1}, \ldots, z_{i}^{1}, \ldots, x_{n}^{1} ; t_{i}^{1}\right) \geq 0 \text { and } f_{i}\left(x_{1}^{2}, \ldots, z_{i}^{2}, \ldots, x_{n}^{2} ; t_{i}^{2}\right) \geq 0 .
$$

From condition (c), we have

$$
\begin{aligned}
& d_{i}\left(z_{i}^{1}, z_{i}^{2}\right) \leq-\frac{1}{m_{i}} f_{i}\left(x_{1}^{1}, \ldots, z_{i}^{1}, \ldots, x_{n}^{1} ; z_{i}^{2}\right)-\frac{1}{m_{i}} f_{i}\left(x_{1}^{1}, \ldots, z_{i}^{2}, \ldots, x_{n}^{1} ; z_{i}^{1}\right), \\
& d_{i}\left(z_{i}^{1}, z_{i}^{2}\right) \leq-\frac{1}{m_{i}} f_{i}\left(x_{1}^{2}, \ldots, z_{i}^{1}, \ldots, x_{n}^{2} ; z_{i}^{2}\right)-\frac{1}{m_{i}} f_{i}\left(x_{1}^{2}, \ldots, z_{i}^{2}, \ldots, x_{n}^{2} ; z_{i}^{1}\right) .
\end{aligned}
$$

Next, adding these two inequalities, using (3.5) and hypothesis (d) follows that

$$
\begin{aligned}
d_{i}\left(z_{i}^{1}, z_{i}^{2}\right) & \leq-\frac{1}{2 m_{i}} f_{i}\left(x_{1}^{1}, \ldots, z_{i}^{1}, \ldots, x_{n}^{1} ; z_{i}^{2}\right)+\frac{1}{2 m_{i}} f_{i}\left(x_{1}^{1}, \ldots, z_{i}^{1}, \ldots, x_{n}^{1} ; t_{i}^{1}\right) \\
& -\frac{1}{2 m_{i}} f_{i}\left(x_{1}^{1}, \ldots, z_{i}^{2}, \ldots, x_{n}^{1} ; z_{i}^{1}\right)-\frac{1}{2 m_{i}} f_{i}\left(x_{1}^{2}, \ldots, z_{i}^{2}, \ldots, x_{n}^{2} ; z_{i}^{1}\right) \\
& +\frac{1}{2 m_{i}} f_{i}\left(x_{1}^{2}, \ldots, z_{i}^{2}, \ldots, x_{n}^{2} ; t_{i}^{2}\right)-\frac{1}{2 m_{i}} f_{i}\left(x_{1}^{2}, \ldots, z_{i}^{1}, \ldots, x_{n}^{2} ; z_{i}^{2}\right) \\
& \leq \frac{L_{i}}{2 m_{i}} d_{i}\left(z_{i}^{2}, t_{i}^{1}\right)+\frac{L_{i}}{2 m_{i}} d_{i}\left(z_{i}^{1}, t_{i}^{2}\right) \\
& -\frac{1}{2 m_{i}} f_{i}\left(x_{1}^{1}, \ldots, z_{i}^{2}, \ldots, x_{n}^{1} ; z_{i}^{1}\right)-\frac{1}{2 m_{i}} f_{i}\left(x_{1}^{2}, \ldots, z_{i}^{1}, \ldots, x_{n}^{2} ; z_{i}^{2}\right) .
\end{aligned}
$$

On the other hand, $z_{i}^{1} \in \gamma\left(x^{1}\right)$ implies that $f_{i}\left(x_{1}^{1}, \ldots, z_{i}^{1}, \ldots, x_{n}^{1} ; z_{i}^{1}\right) \geq 0$. Similarly, we have $f_{i}\left(x_{1}^{2}, \ldots, z_{i}^{2}, \ldots, x_{n}^{2} ; z_{i}^{2}\right) \geq 0$. So it follows, using also condition (e), the previous inequality, (3.3) and (3.4) that

$$
\begin{aligned}
d_{i}\left(z_{i}^{1}, z_{i}^{2}\right) & \leq \frac{L_{i}}{2 m_{i}} d_{i}\left(z_{i}^{2}, t_{i}^{1}\right)+\frac{L_{i}}{2 m_{i}} d_{i}\left(z_{i}^{1}, t_{i}^{2}\right) \\
& -\frac{1}{2 m_{i}} f_{i}\left(x_{1}^{1}, \ldots, z_{i}^{2}, \ldots, x_{n}^{1} ; z_{i}^{1}\right)-\frac{1}{2 m_{i}} f_{i}\left(x_{1}^{2}, \ldots, z_{i}^{1}, \ldots, x_{n}^{2} ; z_{i}^{2}\right) \\
& +\frac{1}{2 m_{i}} f_{i}\left(x_{1}^{1}, \ldots, z_{i}^{1}, \ldots, x_{n}^{1} ; z_{i}^{1}\right)+\frac{1}{2 m_{i}} f_{i}\left(x_{1}^{2}, \ldots, z_{i}^{2}, \ldots, x_{n}^{2} ; z_{i}^{2}\right) \\
& \leq \frac{L_{i}}{m_{i}} H_{i}\left(S_{i}\left(x^{1}\right), S_{i}\left(x^{2}\right)\right)+\frac{L_{i} \varepsilon}{m_{i}}+\frac{\lambda_{i}}{m_{i}} d_{i}\left(z_{i}^{1}, z_{i}^{2}\right) .
\end{aligned}
$$


From here, we get

$$
\left(1-\frac{\lambda_{i}}{m_{i}}\right) d_{i}\left(z_{i}^{1}, z_{i}^{2}\right) \leq \frac{L_{i}}{m_{i}} H_{i}\left(S_{i}\left(x^{1}\right), S_{i}\left(x^{2}\right)\right)+\frac{L_{i} \varepsilon}{m_{i}} .
$$

When $\varepsilon \rightarrow 0$, the inequality becomes

$$
d_{i}\left(z_{i}^{1}, z_{i}^{2}\right) \leq \frac{L_{i}}{m_{i}-\lambda_{i}} H_{i}\left(S_{i}\left(x^{1}\right), S_{i}\left(x^{2}\right)\right),
$$

so $\left.q_{i}=\frac{L_{i}}{m_{i}-\lambda_{i}} \in\right] 0,1[$ as needed.

We mention that sufficient conditions for the non-emptiness of the sets $\Gamma_{i}(x)$ or $T_{i}(x)$ can be given, for instance, by using intersection theorems of Ky Fan type (see [5], [4]).

\section{REFERENCES}

[1] R. P. Agarwal, M. Balaj and D. O'Regan: Variational relation problems in a general setting. Journal of Fixed Point Theory and Applications 18 (2016), 479-493.

[2] M. Balaj: Systems of variational relations with lower semicontinuous set-valued mappings. Carpathian Journal of Mathematics 31 (2015), 269-275.

[3] A. Granas and J. Dugundji: Fixed Point Theory, Springer-Verlag, Berlin, 2003.

[4] D. Inoan: Variational relations problems via fixed points of contraction mappings. Journal of Fixed Point Theory and Applications 19 (2017), 1571-1580.

[5] D. Inoan: Factorization of quasi-variational relations systems. Acta Mathematica Vietnamica 39 (2014), $359-365$.

[6] G. Kassay, J. Kolumbán and Z. Páles: Factorization of Minty and Stampacchia variational inequality systems. European J. Oper. Res. 143 (2002), 377-389.

[7] A. Latif and D. T. Luc: Variational relation problems: existence of solutions and fixed points of contraction mappings. Fixed Point Theory and Applications (2013) Article id. 315, 1-10.

[8] L-J. Lin and Q. H. Ansari: Systems of quasi-variational relations with applications. Nonlinear Anal. 72 (2010), 12101220.

[9] L-J. Lin, M. Balaj and Y. C. Ye: Quasi-variational relation problems and generalized Ekeland's variational principle with applications. Optimization 63 (2014), 1353-1365.

[10] L-J. Lin and S-Y. Wang: Simultaneous variational relation problems and related applications. Computers and Mathematics with Applications 58 (2009), 1711-1721.

[11] D. T. Luc: An abstract problem in variational analysis. J. Optim. Theory Appl. 138 (2008), 65-76.

[12] Y. J. Pu and Z. Yang: Variational relation problem without the KKM property with applications. J. Math. Anal. Appl. 393 (2012), 256-264.

[13] S. Reich: Fixed point of contractive functions. Boll. Un. Mat. Ital. 5 (1972), 26-42.

[14] I. A. Rus, A. Petrusel and G. Petrusel: Fixed Point Theory, Cluj University Press, Cluj-Napoca, 2008.

\section{TECHNiCAL UNIVERSITY OF CLUJ-NAPOCA}

DEPARTMENT OF MATHEMATICS

MEMORANDUMULUI STR. NR. 28, 400114 CLUJ-NAPOCA, ROMANIA

ORCID: 0000-0003-4666-1480

Email address: daniela.inoan@math.utcluj.ro 